Bull. Korean Math. Soc. 47 (2010), No. 5, pp. 1077-1087

DOI 10.4134/BKMS.2010.47.5.1077

\title{
ON RINGS IN WHICH EVERY IDEAL IS WEAKLY PRIME
}

\author{
Yasuyuki Hirano, Edward Poon, and Hisaya Tsutsui
}

\begin{abstract}
Anderson-Smith [1] studied weakly prime ideals for a commutative ring with identity. Blair-Tsutsui [2] studied the structure of a ring in which every ideal is prime. In this paper we investigate the structure of rings, not necessarily commutative, in which all ideals are weakly prime.
\end{abstract}

\section{Introduction}

Anderson-Smith [1] defined a proper ideal $P$ of a commutative ring $R$ with identity to be weakly prime if $0 \neq a b \in P$ implies $a \in P$ or $b \in P$, and then it is proved that every proper ideal in a commutative ring $R$ with identity is weakly prime if and only if either $R$ is a quasilocal ring (possibly a field) whose maximal ideal is square zero, or $R$ is a direct sum of two fields [1, Theorem 8]. On the other hand, Blair-Tsutsui [2] studied the structure of a ring in which every ideal is prime. In this paper we first consider the structure of rings, not necessarily commutative nor with identity, in which all ideals are weakly prime. A necessary and sufficient condition for a ring to have such property is given and several examples to support given propositions are constructed. We then further investigate commutative rings in which every ideal is weakly prime and the structure of such rings under assumptions that generalize commutativity of rings. At the end, we consider the structure of rings in which every right ideal is weakly prime.

\section{Definitions and general results}

Throughout this paper, all rings are associative. By a ring $R$ with identity, we shall mean that $R$ has a multiplicative identity $1 \neq 0$.

By Theorem 3 of Anderson-Smith [1], the following statements are equivalent for an ideal $P$ of a commutative ring $R$ with identity:

(a) $P$ is weakly prime.

(b) For ideals $A$ and $B$ of $R, 0 \neq A B \subseteq P$ implies $A \subseteq P$ or $B \subseteq P$.

Received April 13, 2009; Revised October 1, 2009

2000 Mathematics Subject Classification. 16N60, 16W99.

Key words and phrases. weakly prime. 
For rings that are not necessarily commutative, it is clear that (b) does not imply (a). The standard definition of a prime ideal $P$ for a noncommutative ring $R$ is that for ideals $A$ and $B$ of $R, A B \subseteq P$ implies $A \subseteq P$ or $B \subseteq P$. Accordingly, we define an ideal of a ring $R$ to be weakly prime as follows.

Definition. We define a proper ideal $I$ of a ring $R$ to be weakly prime if $0 \neq J K \subseteq I$ implies either $J \subseteq I$ or $K \subseteq I$ for any ideals $J, K$ of $R$.

Our first proposition is Theorem 1 of Anderson-Smith [1] in a more general setting.

Proposition 1. If $P$ is weakly prime but not prime, then $P^{2}=0$.

Proof. Since $P$ is weakly prime but not prime, there exist ideals $I \nsubseteq P$ and $J \nsubseteq P$ but $0=I J \subseteq P$. But if $P^{2} \neq 0$, then $0 \neq P^{2} \subseteq(I+P)(J+P) \subseteq P$, which implies $I \subseteq P$ or $J \subseteq P$, a contradiction. Hence, $P^{2}=0$.

Proposition 2. Let $P$ be an ideal in a ring $R$ with identity. The following statements are equivalent:

(1) $P$ is a weakly prime ideal.

(2) If $J, K$ are right (left) ideals of $R$ such that $0 \neq J K \subseteq P$, then $J \subseteq P$ or $K \subseteq P$.

(3) If $a, b \in R$ such that $0 \neq a R b \subseteq P$, then $a \in P$ or $b \in P$.

Proof. The implications $(1) \Rightarrow(2)$ and $(2) \Rightarrow(3)$ are easy, but we include their proofs for completeness.

$(1) \Rightarrow(2)$ : Assume (1) holds. Suppose $J, K$ are right (left) ideals of $R$ such that $0 \neq J K \subseteq P$. Let $(J),(K)$ be the ideals generated by $J, K$ respectively. Then $0 \neq(J)(K) \subseteq P$, whence $J \subseteq(J) \subseteq P$ or $J \subseteq(J) \subseteq P$.

$(2) \Rightarrow(3)$ : Assume (2) holds. Suppose $0 \neq a R b \subseteq P$. Since $R$ has an identity, $0 \neq(a R)(b R) \subseteq P$, whence $a \in a R \subseteq P$ or $b \in b R \subseteq P$.

$(3) \Rightarrow(1)$ : Assume (3) holds. Suppose that $J K \subseteq P$ for ideals $J$ and $K$ of $R$, where $K \not \subset P$ and $J \not \subset P$. Let $x \in J \backslash P, y \in K \backslash P, x^{\prime} \in J \cap P$, and $y^{\prime} \in K \cap P$ be arbitrary. Since $x+x^{\prime}, y+y^{\prime} \notin P$, we must have $0=\left(x+x^{\prime}\right) R\left(y+y^{\prime}\right)$. Considering all combinations where $x^{\prime}$ and/or $y^{\prime}$ equal zero shows that $0=x y=$ $x y^{\prime}=x^{\prime} y=x^{\prime} y^{\prime}$, and hence $J K=0$.

We are interested in the structure of rings in which every ideal is weakly prime. Note that by definition, a weakly prime ideal is a proper ideal of a ring. It is therefore not possible that every ideal of a ring is a weakly prime ideal. However, a ring whose zero ideal is prime is called a prime ring. In this sense, every ring is a weakly prime ring since the zero ideal is always weakly prime. We may therefore say that every ideal of a ring is weakly prime when every proper ideal of the ring is a weakly prime ideal.

If $R^{2}=0$, then it is evident that every ideal of $R$ is weakly prime. In particular, if an ideal $I$ of a ring $R$ is weakly prime but not a prime ideal, then every ideal of $I$ as a ring is weakly prime by Proposition 1 . 
Proposition 3. Every ideal of a ring $R$ is weakly prime if and only if for any ideals $I$ and $J$ of $R, I J=I, I J=J$, or $I J=0$.

Proof. Suppose that every ideal of $R$ is weakly prime. Let $I, J$ be ideals of $R$. If $I J \neq R$, then $I J$ is weakly prime. If $0 \neq I J \subseteq I J$, then we have $I \subseteq I J$ or $J \subseteq I J$, that is, $I=I J$ or $J=I J$. If $I J=R$, then we have $I=J=R$ whence $R^{2}=R$.

Conversely, let $K$ be any proper ideal of $R$ and suppose that $0 \neq I J \subseteq K$ for ideals $I$ and $J$ of $R$. Then we have either $I=I J \subseteq K$ or $J=I J \subseteq K$.

Corollary 1. Let $R$ be a ring in which every ideal of $R$ is weakly prime. Then for any ideal $I$ of $R$, either $I^{2}=I$ or $I^{2}=0$.

Example 1. Let $F$ be a field and $R=F \oplus F \oplus F$. Then every ideal of $R$ is idempotent but the ideal $I=F \oplus 0 \oplus 0$ is evidently not weakly prime, showing that the converse of Corollary 1 is false.

Suppose that a ring $R$ with identity has a maximal ideal $M$ and $M^{2}=0$. Thus, the product of any two ideals contained in $M$ is zero. It is evident that every proper ideal of $R$ is contained in $M$, and for any ideal $I, I R=R I=I$. Hence, every ideal of $R$ is weakly prime. In this case, notice that $M$ is the only prime ideal of $R$.

Corollary 1 in particular yields that if a ring $R$ has the property that every ideal is weakly prime, then either $R^{2}=R$, or $R^{2}=0$. Notice that $R^{2}$ is neither 0 nor $R$ in the example given below.

Example 2. Let $S$ be a ring such that $S^{2}=0$, and let $F$ be a field. Then the ring $R=F \oplus S \oplus S$ with component-wise addition and multiplication has a maximal ideal $M=0 \oplus S \oplus S$ and $M^{2}=0$. However, $I=F \oplus 0 \oplus S$ is not weakly prime since $0 \neq(F \oplus S \oplus 0)^{2} \subseteq I$.

If a ring $R$ satisfying $R^{2}=R$ has a maximal ideal $M$ and $M^{2}=0$, then every proper ideal of $R$ is contained in $M$. However, it is possible that $M R \neq M$. Thus, such a ring does not necessarily have the property that every ideal is weakly prime as the following example shows.

Example 3. Let $F$ be a field and $S=\left\{\left[\begin{array}{lll}0 & a & b \\ 0 & c & d \\ 0 & 0 & 0\end{array}\right] \mid a, b, c, d \in F\right\}$. Then $S$ has a unique maximal ideal $L=\left\{\left[\begin{array}{lll}0 & a & b \\ 0 & 0 & d \\ 0 & 0 & 0\end{array}\right] \mid a, b, d \in F\right\}$. Let $N=\left\{\left[\begin{array}{lll}0 & 0 & b \\ 0 & 0 & 0 \\ 0 & 0 & 0\end{array}\right] \mid b \in F\right\}$. Consider the factor ring $R=S / N$. While $R^{2}=R$ and $M=L / N$ is a maximal ideal whose square is zero, the proper ideals $R M$ and $M R$ are not weakly prime.

Proposition 4. If every ideal of a ring $R$ is weakly prime and $R^{2}=R$, then $R$ has at most two maximal ideals.

Proof. Suppose that $R$ has more than two distinct maximal ideals. Let $M_{1}$, $M_{2}$, and $M_{3}$ be three distinct maximal ideals. Then $M_{1} M_{2} \neq 0$. Thus we have 
$0 \neq M_{1} M_{2} \subseteq M_{1} \cap M_{2}$. But this implies either $M_{1} \subseteq M_{2}$ or $M_{2} \subseteq M_{1}$, a contradiction.

The following example shows that the condition $R^{2}=R$ in Proposition 4 cannot be dropped.

Example 4. Let $R$ be the unique maximal ideal of $\mathbb{Z}_{4}$. Then $S=R \oplus R \oplus R$ is an example of a ring all of whose ideals are weakly prime and having more than 2 maximal ideals.

Proposition 5. Suppose that every ideal of a ring $R$ is weakly prime. If $R$ has two maximal ideals, then their product is zero. Furthermore, if $R$ has an identity element, then $R$ is a direct sum of two simple rings.

Proof. Suppose that $R$ has two distinct maximal ideals $M_{1}$ and $M_{2}$. Then since $M_{1} \cap M_{2}$ is weakly prime and $M_{1} M_{2} \subseteq M_{1} \cap M_{2}$, we must have $M_{1} M_{2}=0$, and similarly $M_{2} M_{1}=0$.

If $R$ has an identity element, then $M_{1} \cap M_{2}=\left(M_{1} \cap M_{2}\right) R=\left(M_{1} \cap M_{2}\right)\left(M_{1}+\right.$ $\left.M_{2}\right) \subseteq M_{2} M_{1}+M_{1} M_{2}=0$, and this implies that $R \simeq R / M_{1} \oplus R / M_{2}$.

Suppose that every ideal of a ring $R$ is weakly prime. By Proposition 1 and Corollary 1 , any nontrivial idempotent ideal of $R$ is a prime ideal. Recall that the intersection of all prime ideals of a $\operatorname{ring} R$ is called the prime radical of $R$. Hereafter, we denote the prime radical of $R$ by $P(R)$, and the sum of all ideals whose square is zero by $N(R)$.

Theorem 1. Suppose that every ideal of a ring $R$ is weakly prime and $R^{2}=R$. Then $P(R)=N(R)$ and $(P(R))^{2}=(N(R))^{2}=0$.

Proof. Take $a_{1}, a_{2} \in N(R)$. Then there are finitely many square-zero ideals $I_{1}, I_{2}, \ldots, I_{m}$ such that $a_{1}, a_{2} \in I_{1}+I_{2}+\cdots+I_{m}$. Since $I_{j}^{2}=0$ for each $j$, $\left(I_{1}+I_{2}+\cdots+I_{m}\right)^{k}=0$ for some $k$ but then $\left(I_{1}+I_{2}+\cdots+I_{m}\right)^{2}=0$ by Corollary 1. Hence, $(N(R))^{2}=0$. This implies that if $P$ is any prime ideal of $R, N(R) \subseteq P$ and consequently, $N(R) \subseteq P(R)$. We should note that $R$ contains at least one prime ideal. Indeed, if $R$ contains a nonzero idempotent ideal, then by the remark above, it must be prime. If every ideal is nilpotent, then since $R^{2}=R, N(R) \neq R$ is a prime ideal.

If $P(R)$ is not a prime ideal, then $(P(R))^{2}=0$. This implies that $P(R) \subseteq$ $N(R)$ by the definition of $N(R)$, and hence the result follows.

Suppose $P(R)$ is a prime ideal. In this case, we will show that $N(R)$ must also be prime. This implies that $P(R) \subseteq N(R)$ by the definition of $P(R)$, and hence the result follows. To show that $N(R)$ is also a prime ideal, note first that since $N(R) \subseteq P(R)$, every ideal containing $P(R)$ is a non-nilpotent ideal and thus they are all prime ideals. This implies that ideals of $R / P(R)$ are linearly ordered by Theorem 1.2 of Blair-Tsutsui [2] and therefore the prime ideals of $R$ are linearly ordered. 
Suppose now that $I J \subseteq N(R)$ for ideals $I$ and $J$ of $R$. Since $N(R)$ is weakly prime, we have $J \subseteq N(R)$ or $I \subseteq N(R)$ if $I J \neq 0$. Suppose $I J=0$. If $I^{2}=0$ or $J^{2}=0$, then $J \subseteq \bar{N}(R)$ or $I \subseteq \bar{N}(R)$. If both $I$ and $J$ are not square zero, then they are prime ideals. But then either $I=I^{2} \subseteq I J=0$ or $J=J^{2} \subseteq I J=0$, a contradiction. This shows that $N(R)$ is a prime ideal.

Corollary 2. Suppose that every ideal of a right Noetherian ring $R$ with identity is weakly prime and $R^{2}=R$. Then $P(R)=N(R)=J(R)$ and $(J(R))^{2}=(P(R))^{2}=(N(R))^{2}=0$, where $J(R)$ is the Jacobson radical of $R$.

Proof. If $(J(R))^{2}=J(R)$, then $J(R)=0$ by Nakayama's lemma. Since $P(R) \subseteq$ $J(R)$, the result follows. If $(J(R))^{2}=0$, then $J(R) \subseteq P$ for every prime ideal $P$ in $R$. Hence $J(R) \subseteq P(R)$.

Note that for a ring $R$ in which every ideal is weakly prime, in general it is possible that $P(R)=N(R) \neq J(R)$, where $J(R)$ is the Jacobson radical of $R$. See $\S 5$ an example of Blair-Tsutsui [2].

Corollary 3. Suppose that every ideal of a ring $R$ is weakly prime. Then every nonzero ideal of $R / N(R)$ is prime.

Corollary 4. Suppose that every ideal of a ring $R$ is weakly prime. Then $(N(R))^{2}=0$ and every prime ideal contains $N(R)$. There are three possibilities: (a) $N(R)=R$.

(b) $N(R)=P(R)$ is the smallest prime ideal and all other prime ideals are idempotent and prime ideals are linearly ordered. If $N(R) \neq 0$, then it is the only non-idempotent prime ideal.

(c) $N(R)=P(R)$ is not a prime ideal. In this case, there exist two nonzero minimal prime ideals $J_{1}$ and $J_{2}$ with $N(R)=J_{1} \cap J_{2}$ and $J_{1} J_{2}=J_{2} J_{1}=0$. All other ideals containing $N(R)$ also contain $J_{1}+J_{2}$ and they are linearly ordered.

Proof. If $R^{2}=0$, then $N(R)=R$. So clearly $(N(R))^{2}=0$, and there are no prime ideals.

If $R^{2}=R$, then by Theorem $1, P(R)=N(R)$ and $(P(R))^{2}=(N(R))^{2}=0$. By the definition of $P(R)$, every prime ideal contains $N(R)=P(R)$.

If $N(R)=P(R)$ is prime, it is evidently the smallest prime ideal and all other prime ideals are idempotent. Further, in our proof of Theorem 1, we observed that all prime ideals of $R$ are linearly ordered.

If $N(R)=P(R)$ is not a prime ideal, then since every ideal of $R / N(R)$ except 0 is prime, the ideals of $R / N(R)$ are not linearly ordered by Theorem 1.2 of Blair-Tsutsui [2]. Thus, by Theorem 2.1 of Tsutsui [4], there exist two nonzero minimal prime ideals $J_{1}$ and $J_{2}$ with $N(R)=J_{1} \cap J_{2}$. Moreover, all other ideals containing $N(R)$ also contain $J_{1}+J_{2}$ and they are linearly ordered. Since $N(R)=J_{1} \cap J_{2}$ is weakly prime, we must have $J_{1} J_{2}=J_{2} J_{1}=0$. Otherwise, $J_{1} J_{2}, J_{2} J_{1} \subseteq J_{1} \cap J_{2}$ implies either $J_{1} \subseteq J_{1} \cap J_{2} \subseteq J_{2}$ or $J_{2} \subseteq J_{1} \cap J_{2} \subseteq J_{1}$, giving a contradiction. 
Example 5. Let $R$ be a ring and $M$ an $R$-bimodule. Define

$$
R * M=\{(r, m) \mid r \in R, m \in M\}
$$

with component-wise addition and multiplication $(r, m)(s, n)=(r s, r n+m s)$. Then $R * M$ is a ring whose ideals are precisely of the form $I * N$ where $I$ is an ideal of $R$ and $N$ is a submodule (a bimodule) of $M$ containing $I M$ and $M I$.

(a) Let $R$ be a prime ring that contains exactly one nonzero proper ideal $P$. For example, the ring of linear transformations of a vector space $V$ over a field $F$ where $\operatorname{dim}_{F} V=\aleph_{0}$ has such a property. Then every ideal of $S_{1}=R * P$ is weakly prime: the maximum ideal $P_{1}=P * P$ is idempotent and the nonzero minimal ideal $P_{2}=0 * P$ is nilpotent, both of which are prime.

(b) Every ideal of $S_{2}=S_{1} * P_{2}$ is weakly prime: The maximum ideal $Q_{1}=$ $P_{1} * P_{2}$ is idempotent and the three nonzero nilpotent ideals are $Q_{2}=P_{2} * P_{2}$, $Q_{3}=0 * P_{2}$, and $Q_{4}=P_{2} * 0$.

(c) If we redefine the multiplication above as $(r, m)(s, n)=(r s, r n+m s+$ $m n$ ), then $S_{1}$ in (a) has an additional minimal ideal $P_{3}=\{(p,-p) \mid p \in P\}$. In this case, $N\left(S_{1}\right)=P_{3} \cap P_{2}=0$.

We do not know of an example of the case (c) in Corollary 4 where $N(R) \neq 0$.

\section{Commutative rings and generalizations thereof}

We now consider the structure of rings in which every ideal is weakly prime under the assumption of the ring being commutative or with commutative-like conditions.

Proposition 6. Let $R$ be a commutative ring in which every ideal is weakly prime. If $R^{2}=R$, then $R$ has a maximal ideal.

Proof. If there exists a nonzero idempotent proper ideal $I$, then every ideal containing $I$ must be idempotent and hence prime. This implies that every ideal of $R / I$ is prime. By Theorem 1.3 of Blair-Tsutsui [2], this implies that $I$ is a maximal ideal of $R$. If all proper ideals are nilpotent, then since $R^{2}=R$, $N(R) \neq R$ is the maximal ideal.

We note that a commutative ring $R$ with the property $R^{2}=R$ does not necessarily have a maximal ideal. For example, if a commutative ring $S$ has a unique nonzero maximal ideal $M$ and $M^{2}=M$, then $M$ as a ring cannot have a maximal ideal.

The following corollary is a direct consequence of Proposition 4 and Proposition 6 .

Corollary 5. Let $R$ be a commutative ring all of whose ideals are weakly prime. Suppose that $R^{2}=R$. Then $R$ has either a unique maximal ideal or exactly two maximal ideals. 
Theorem 2. Let $R$ be a commutative ring all of whose ideals are weakly prime. Suppose that $R^{2}=R$.

(1) If $R$ has a unique maximal ideal $M$, then $M^{2}=0$.

(2) If $R$ has two maximal ideals $M$ and $N$, then $M N=0$.

Proof. (1) Suppose that $R$ has a unique maximal ideal $M$ and $M^{2}=M \neq 0$. If $N(R)$ is prime, then by Corollary $3, R / N(R)$ is a commutative ring $R$ all of whose ideals are prime. Hence $R / N(R)$ is a field and we must have $N(R)=$ $M$, a contradiction to the assumption that $M^{2}=M \neq 0$. If $N(R)$ is not a prime ideal, then there exist two nonzero minimal prime ideals $J_{1}$ and $J_{2}$ with $N(R)=J_{1} \cap J_{2}$ by Corollary 4 . But then since $R / J_{1}$ and $R / J_{2}$ must both be fields, we have $N(R)=J_{1} \cap J_{2}=M$ and this is also a contradiction.

(2) Suppose that $R$ has two maximal ideals $M$ and $N$ such that $M N \neq 0$. Then since $M N$ is weakly prime, we have either $N \subseteq M N \subseteq M$ or $M \subseteq$ $M N \subseteq N$, a contradiction.

Proposition 7. Let $R$ be a commutative ring all of whose ideals are weakly prime. Suppose that $R^{2}=R$. Then every proper ideal is contained in a maximal ideal.

Proof. $R$ has either a unique maximal ideal whose square is zero or exactly two maximal ideals whose product is zero.

Let $M$ be a maximal ideal. Suppose that $L$ is a proper ideal of $R$ and $L \not \subset M$. Since $R^{2}=R, M$ is a prime ideal and hence $L^{2} \not \subset M$. This implies that $L^{2} \neq 0$ and thus $L$ is a prime ideal. Therefore, if $M^{2}=0$, we get $L=M$.

If $M^{2}=M$, then there exists another maximal ideal $N$ and $M N=0 \subseteq L$. This implies $N=L$. We now conclude that every ideal in $R$ is contained in a maximal ideal of $R$.

Corollary 6. Let $R$ be a commutative ring and suppose that every ideal of $R$ is weakly prime. If $R^{2}=R$, then $R$ has an identity element.

Proof. We show that if a commutative ring $R$ satisfies the following conditions, then $R$ has an identity element:

(a) $R^{2}=R$,

(b) every proper ideal is contained in a maximal ideal, and

(c) $R$ has a finite number of maximal ideals $M_{1}, M_{2}, \ldots, M_{n}$.

Choose $x \in R$ such that $x \notin M_{j}$ for any $J$. Let $(x)=\{x R+n x \mid n \in \mathbb{Z}\}$. If $x R \subseteq M_{j}$, then $R=R^{2}=\left(M_{j}+(x)\right)^{2} \subseteq M_{j}$, a contradiction. Hence $x R=R$ and consequently, $R$ has an identity element.

Corollary 7. Let $R$ be a commutative ring all of whose ideals are weakly prime. Then one of the following holds:
(a) $R^{2}=0$,
(b) $R$ is a ring with identity and with a square zero maximal ideal $M$, or
(c) $R$ is a direct sum of two fields. 
For the case (b) in Corollary 7, the following theorem further determines the structure of $R$.

Theorem 3. Let $R$ be a commutative ring with a square-zero maximal ideal $M$ and $R^{2}=R$. If $(\operatorname{ch}(R / M)=0$, then $R$ is isomorphic to $(R / M) * M$ (as defined in Example 5).

Proof. Let $x \notin M$ and $(x)=\{x R+n x \mid n \in \mathbb{Z}\}$. Then since $M^{2}=0, R=$ $R^{2}=(M+(x))^{2} \subseteq x R$ and this implies that $R$ has an identity element. Thus, in particular, any element not in $M$ is invertible.

Let $\pi: R \rightarrow R / M$ be the canonical projection and write $\pi(r)=\bar{r}$. We identify $n 1 \in R$ with $n \in \mathbb{Z}$; since $\operatorname{ch}(R / M)=0$, we have $n \in M$ if and only if $\bar{n}=\overline{0}$ if and only if $n=0$. In particular, any $0 \neq n \in \mathbb{Z}$ is invertible.

Note that if $E$ is a subfield of $R / M$ and $\psi: E \rightarrow R$ is a homomorphism satisfying $\pi \circ \psi=\left.i d\right|_{E}$, then the map $\varphi: E * M \rightarrow R$ given by $\varphi((\bar{x}, m))=$ $\psi(\bar{x})+m$ is a monomorphism. Thus, it suffices to show that such a map $\psi$ exists for $E=R / M$ (in this case $\varphi$ is also onto); we proceed in stages, defining $\psi$ on successively larger subfields $E \subseteq R / M$.

Step 1: Let $K$ be the base field for $R / M$. Define $\psi: K \rightarrow R$ by $\psi\left(\bar{a} \bar{b}^{-1}\right)=$ $a b^{-1}$ for any $a, 0 \neq b \in \mathbb{Z}$. Then $\psi$ is a homomorphism and clearly $\pi \circ \psi=\left.i d\right|_{K}$.

Step 2: Let $\pi(T)$ be a transcendence base for $R / M$ over $K$ and write $F=$ $K(\pi(T))$. Then any nonzero element in the subring generated by $\psi(K)$ and $T$ is invertible. Let $R_{T}$ denote the field in $R$ generated by $\psi(K)$ and $T$. Define $\psi(\bar{t})=t$ for any $t \in T$. Then $\psi$ extends to an isomorphism from $F$ to $R_{T}$ and $\left.\pi \circ \psi\right|_{F}=\left.i d\right|_{F}$.

Step 3: Let $A \subset R / M$ be an algebraic set over $F$ such that $R / M=F(A)$. Well-order $A$.

Write $F_{\bar{b}}$ for $F(\{\bar{a} \in A \mid \bar{a}<\bar{b}\})$ and $\tilde{F}_{\bar{b}}$ for $F(\{\bar{a} \in A \mid \bar{a} \leq \bar{b}\})$. We extend $\psi$ to $R / M$ recursively. Suppose there exists an extension $\psi: F_{\bar{b}} \rightarrow R$ which is a homomorphism satisfying $\left.\pi \circ \psi\right|_{F_{\bar{b}}}=\left.i d\right|_{F_{\bar{b}}}$. It suffices to extend $\psi$ to a homomorphism from $\tilde{F}_{\bar{b}}$ to $R$ satisfying $\left.\pi \circ \psi\right|_{\tilde{F}_{\bar{b}}}=\left.i d\right|_{\tilde{F}_{\bar{b}}}$.

The case $\bar{b} \in F_{\bar{b}}$ is trivial, so suppose $\bar{b} \notin F_{\bar{b}}$. Let $p \in F_{\bar{b}}[x]$ be the minimum polynomial of $\bar{b}$.

If $p(x)=\sum_{k=1}^{n} \bar{c}_{k} x^{k}$, define $p_{\psi}(x)=\sum_{k=1}^{n} \psi\left(\bar{c}_{k}\right) x^{k}$. Fix $u \in \pi^{-1}(\bar{b})$. If $p_{\psi}^{\prime}(u) \in M$, then $p^{\prime}(b)=\pi\left(p_{\psi}^{\prime}(u)\right)=\overline{0}$, and this contradicts the minimality of $p$. So $p_{\psi}^{\prime}(u)$ is invertible.

Define $m=-p_{\psi}(u)\left[p_{\psi}^{\prime}(u)\right]^{-1} \in M$. Then $p_{\psi}(u+m)=0$, so $v=u+m$ satisfies $p_{\psi}(v)=0$.

Define $\psi(\bar{b})=v$, and more generally, $\psi\left(\sum_{k=1}^{n} \bar{a}_{k} \bar{b}^{k}\right)=\sum_{k=1}^{n} \psi\left(\bar{a}_{k}\right) v^{k}$ for all $\bar{a}_{k} \in F_{\bar{b}}$. Then $\psi$ extends to a homomorphism from $\tilde{F}_{\bar{b}}$ to $R$ and $\left.\pi \circ \psi\right|_{\tilde{F}_{\bar{b}}}=$ $\left.i d\right|_{\tilde{F}_{\bar{b}}}$. 
Using the same proof, the result also holds for $\operatorname{ch}(R / M)=p$ if $p R=0$ and $R / M$ is separable over $F$. In general, however, the theorem is false if $\operatorname{ch}(R / M)=p \neq 0$.

Example 6. (a) Let $R=Z_{p^{2}}$ where $p$ is prime. Then $R$ has maximal ideal $M=p R \neq 0$ but $p(R / M * M)=0$.

(b) Let $R=\left[\begin{array}{cc}F & F \\ 0 & 0\end{array}\right]$ where $F$ is a field. Then $R^{2}=R \neq 0$, and every ideal of $R$ is weakly prime but $R$ does not contain an identity element.

As a natural generalization of commutative rings, we next consider polynomial identity rings.

Theorem 4. Let $R$ be a ring with identity that satisfies a polynomial identity. If every ideal of $R$ is weakly prime, then one of the following holds:

(a) $R / P(R)$ is a finite dimensional central simple algebra.

(b) $R$ is a direct sum of two finite dimensional central simple algebras.

Proof. If $P(R)$ is a prime ideal, then every ideal of $R / P(R)$ is prime by Theorem 1 and Corollary 3 and hence (a) holds by Theorem 3.3 of Blair-Tsutsui [2].

If $P(R)$ is not a prime ideal, then by Corollary 4 (c), there exist two nonzero minimal prime ideals $J_{1}$ and $J_{2}$. Since every ideal of $R / J_{1}$ is prime and since $R / J_{1}$ satisfies a polynomial identity, $R / J_{1}$ is a finite dimensional central simple algebra by Theorem 3.3 of Blair-Tsutsui [2]. By the same reason, $R / J_{2}$ is a finite dimensional central simple algebra. Since $R$ has two maximal ideals $J_{1}$ and $J_{2}, R \simeq R / J_{1} \oplus R / J_{2}$ by Proposition 5 .

More general than the class of PI-rings is the class of fully bounded rings. Using Theorem 3.4 of Blair-Tsutsui [2], essentially the same proof as given above yields the following theorem.

Theorem 5. Let $R$ be a ring with identity in which every ideal is weakly prime. If $R$ is a right fully bounded, right Noetherian ring, then one of the following holds:

(a) $R / P(R)$ is a simple Artinian ring.

(b) $R$ is a direct sum of two simple Artinian rings.

\section{Rings in which every right ideal is weakly prime}

Recall that a proper right ideal $I$ of a ring $R$ is called prime if $J K \subseteq I$ implies either $J \subseteq I$ or $K \subseteq I$ for any right ideals $J, K$ of $R$.

Definition. We define a proper right ideal $I$ of a $\operatorname{ring} R$ to be weakly prime if $0 \neq J K \subseteq I$ implies either $J \subseteq I$ or $K \subseteq I$ for any right ideals $J, K$ of $R$.

For a ring $R$ that is not square zero, Koh [3] showed that $R$ is simple and $a \in a R$ for all $a \in R$ if and only if every right ideal of $R$ is prime. We now briefly consider the structure of rings in which every right ideal is weakly prime. Considering the commutative case, it is evident that such rings need 
not be simple. Example 6(b) gives an example of a $\operatorname{ring} R=R^{2}$ in which every right and left ideal is weakly prime.

Unlike the case of weakly prime two sided ideals, there exists a nonzero idempotent weakly prime right ideal that is not prime. For example, if we let $R=\left\{\left[\begin{array}{ll}a & b \\ 0 & c\end{array}\right] \mid a, b, c \in F\right\}$, then $K=\left\{\left[\begin{array}{ll}0 & 0 \\ 0 & t\end{array}\right] \mid t \in F\right\}$ is a weakly prime right ideal and $K^{2}=K \neq 0$. But $K$ is not a prime right ideal.

We conclude this paper with the following theorem, which is a generalization of Corollary 7.

Theorem 6. Suppose that every right ideal of a ring $R$ is weakly prime. Then one of the following holds:

(a) $R^{2}=0$.

(b) $R$ has a square zero maximal ideal.

(c) $R$ is a direct sum of two division rings.

To prove this, we will use the following folk theorem. It is undoubtedly well-known, but we include a proof for the sake of completeness.

Proposition 8. Let $R$ be a nonzero ring, and suppose $a R=R$ for all nonzero $a \in R$. Then $R$ is a division ring.

Proof. First note that $R$ has no zero divisors. For if $a, b \neq 0$ and $a b=0$, then $0=a b R=a R=R$, a contradiction.

Fix $a \neq 0$. By hypothesis, there exists $e \in R$ so that $a e=a$. Then $(a e-e a)^{2}=0$, so $a e=e a$. Thus $e a r=a r$ for all $r \in R$; since $a R=R, e$ is a left-identity.

Since $e^{2}=e$, we have $x e^{2}=x e$ for all $x \in R$. Thus $(x e-x) e=0$, whence $x e=x$ and $e$ is a two-sided identity.

Since $x R=R$ for all nonzero $x$, it is clear that all nonzero elements of $R$ are units. Hence $R$ is a division ring.

Proof of Theorem 6. Assume the hypothesis, so Corollary 4 applies. Suppose $R^{2} \neq 0$.

If $N(R)$ is prime, then every right ideal of $R / N(R)$ is prime, so $R / N(R)$ is simple by [3]. Thus $N(R)$ is the square zero maximal ideal.

If $N(R)$ is not prime, then there exist two minimal prime ideals $I, J$ with $N(R)=I \cap J$ and $I J=J I=0$. Again, every right ideal of $R / I$ and $R / J$ is prime, so $I, J$ are maximal ideals and $R=I+J$. We must show that $I \cap J=0$ and that $I, J$ are division rings.

Let $b \in J \backslash I$. Note that $b J \neq 0$ (If $b J=0$, then the left annihilator of $J$ must contain the ideal generated by $b$, namely $J$. But then $J^{2}=0$, a contradiction). Since $(I+b J) J=I J+b J^{2}=0+b J \neq 0$, either $I+b J \subseteq b J \subseteq J$ (contradicting $I \nsubseteq J)$ or $J \subseteq b J \subseteq J$. Hence $r J=J$ for all $r \in J \backslash I$. By Proposition 8, $J / N(R)$ is a division ring, as is $I / N(R)$ by symmetry.

Suppose, by way of contradiction, that $N(R)$ contains an element $n \neq 0$. Since $N(R) \subset J$, there exists $c \in J$ such that $b c=n \neq 0$. Since $J I=0$ we 
must have $c \in J \backslash I$. But $N(R) \subset I$ implies $0=n J=b c J=b J=J$, giving the desired contradiction. So $I \cap J=N(R)=0$, completing the proof.

\section{References}

[1] D. D. Anderson and E. Smith, Weakly prime ideals, Houston J. Math. 29 (2003), no. 4, $831-840$.

[2] W. D. Blair and H. Tsutsui, Fully prime rings, Comm. Algebra 22 (1994), no. 13, 53895400 .

[3] K. Koh, On one sided ideals of a prime type, Proc. Amer. Math. Soc. 28 (1971), 321-329.

[4] H. Tsutsui, Fully prime rings. II, Comm. Algebra 24 (1996), no. 9, 2981-2989.

YASUYUKI HIRANO

Department of Mathematics

NARUto University of Education

NARUTO, JAPAN

E-mail address: yahirano@naruto-u.ac.jp

EDWARD POON

Department of Mathematics

Embry-Riddle Aeronautical University

PrescotT, AZ USA

E-mail address: edward.poon@erau.edu

Hisaya Tsutsui

Department of Mathematics

Embry-Riddle Aeronautical University

Prescott, AZ USA

E-mail address: hisaya.tsutsui@erau.edu 